\title{
RDUS
}

Revue de DROIT

UNIVERSITÉ DE SHERBROOKE

Titre : $\quad$ LES SITUATIONS DE FAIT EN DROIT DES SOCIÉTÉS DE L'OHADA

Auteur(s) : $\quad$ François BIBOUM BIKAY

Revue : $\quad$ RDUS, 2013, volume 43, numéro 3

Pages: $\quad 827-865$

ISSN : $\quad 0317-9656$

Éditeur: $\quad$ Université de Sherbrooke. Faculté de droit.

URI : $\quad$ http://hdl.handle.net/11143/10226

DOI : $\quad$ https://doi.org/10.17118/11143/10226 
Page vide laissée intentionnellement. 


\title{
ARTICLE
}

\section{LES SITUATIONS DE FAIT EN DROIT DES SOCIÉTÉS DE L'OHADA}

\author{
par François BIBOUM BIKAY*
}

L'ensemble des disciplines $d u$ droit connaît le phénomène des situations de fait. Ces dernières constituent des cas de malformation des institutions promues et protégées par le droit. En réaction, celui-ci les répugne souvent en les privant d'effets. Mais la rencontre des situations de fait avec le droit des sociétés vient bouleverser cet état de choses. En effet, non seulement elles y prolifèrent, mais encore elles ne sont pas systématiquement combattues comme dans les autres disciplines juridiques. Cette étude ambitionne donc de montrer comment le droit des sociétés, en s'appuyant sur les situations de fait, atteint son efficacité. La problématique est alors orientée vers le point de savoir qu'elle est la place qu'occupent les situations de fait dans le droit des sociétés. La réponse en est donnée à travers le constat de leur acceptation par la discipline et à travers l'examen de leur contribution à l'efficacité de cette dernière.

De facto situations may be encountered in all fields of law. They tend to deform institutions promoted and protected by law. In reaction, the law rejects them by depriving them of legal effect. However, these encounters between de facto situations and company law in particular do not adhere to the principle of rejection. Not only is there a proliferation of these situations in company law, they are not systematically opposed as they would be in other legal branches. The writer seeks to illustrate how company law, by embracing de facto legal situations, has in fact become more effective. The article then goes on to explain why situations of fact have been openly recognized in company law. The answer may be found in the acceptance and in the contribution of situations of fact to the efficacy of company law.

\footnotetext{
* Docteur en droit privé, assistant à la Faculté des sciences juridiques et politiques de 1'Université de Douala.
} 


\section{SOMMAIRE}

INTRODUCTION

\section{§ I- L'accueil des situations de fait par le droit des}

A- Les groupements d'affaires sans personnalité

morale

1- La société de fait

a- $\quad$ La prise en compte de la volonté

des associés de fait.

b- $\quad$ L'office décisif du juge dans la

qualification de la société de fait.

2- Les autres groupements sans personnalité morale

a- La société en participation

b- La société avant son immatriculation......843

B- L'administration des sociétés sans mandat

1 - Les critères de la direction de fait tenant à la personne

2- Les critères de la direction de fait tenant aux actes

§ II- La contribution des situations de fait à l'efficacité du droit des sociétés 849

A- L'efficacité atteinte par la protection de la société et des parties

1- La protection de la société 850

a- La protection de la société dans sa réalité existentielle

b- La protection de la société dans sa réalité patrimoniale

2- La protection des parties

B- L'efficacité atteinte par la protection des tiers

1- L'existence d'une action en reconnaissance de société de fait au profit du tiers 860

2- L'application aux associés de fait de la solidarité de la société en nom collectif 


\section{INTRODUCTION}

Les juristes ont la coutume d'opposer les faits au droit ${ }^{1}$. Pourtant, la distance entre les deux catégories n'est pas facile à matérialiser. On peut définir les faits comme des évènements soit naturels, soit de l'homme qui n'ont pas un caractère juridique ${ }^{2}$. À partir de là, il est compréhensible que la catégorie des faits juridiques renvoie à ceux pour lesquels le droit a prévu des conséquences juridiques indépendamment de la volonté humaine ${ }^{3}$. Ainsi, une situation est de pur fait lorsqu'on ne peut pas lui attacher des conséquences de droit. C'est alors là que réside la difficulté de la ligne de démarcation entre le fait et ce qui est juridique. Comme cela a été justement remarqué, aucun fait n'est pour le juriste inséparable du droit; il est même une réalité que les juristes, lorsqu'ils veulent disqualifier une situation juridique, la réduisent presque toujours, par un procédé normatif, en un simple fait ${ }^{4}$. Cela veut dire en fait que telle situation est un

1. Voir Maurice TANCELIN, Sources des obligations : l'acte juridique légitime, vol. 1, Montréal, Wilson \& La fleur, 1993, p. 12. Dans la recherche d'un critère de distinction entre les notions d'acte juridique et de fait juridique auxquels il préfère substituer celles d'acte juridique légitime et acte juridique illégitime, cet auteur est amené à constater que "[d]ans l'usage juridique, le mot fait est antonyme du mot droit. En l'employant également comme antonyme d'acte, on crée un risque de confusion entre les mots acte et droit "; Raymond MARTIN, "Le fait et le droit ou les parties et le juge ", (1974) I JCP G., 2625; Gabriel MARTY, La distinction du fait et du droit, essai sur le pouvoir de contrôle de la Cour de cassation sur les juges du fait, thèse de doctorat, Toulouse, Sirey, 1929.

2. Les auteurs préfèrent celles selon lesquelles le fait désigne une circonstance qui tombe sous l'un des cinq sens. Voir par exemple Jean Gatsi, Nouveau Dictionnaire Juridique, 2e éd., Douala, Presses Universitaires Libres, 2009, p. 146; Serge BRAUDO, Dictionnaire du droit privé français, en ligne : <www.dictionnaire-juridique.com> (consulté le 12 mars 2012). Malgré cette posture érudite, il jaillit une idée commune que le fait décrit toute situation à l'état brut de sa considération. À l'égard du droit, elle n'est qu'un fait simple, pour produire des effets juridiques, il faut une qualification en fait juridique par exemple.

3. Raymond Guillien et Jean Vincent (dir), Lexique des termes juridiques, Paris, Dalloz, 2007, p. 301.

4. Bernard S. JACKSON, Law, Fact and Narrative Coherence, Merseyside, Deborah Charles, 1988, p. 16; François RigAuX, "L'opacité du fait face à 
fait parce que le droit la répute comme telle, mais inversement aussi, la situation de droit n'apparaît que comme un ensemble de faits reconnus par le droit ${ }^{5}$. Il s'en suit une telle imbrication qui frise la fongibilité, l'homogénéité des deux phénomènes.

Toutefois, l'opposition n'est pas moins justifiée, tant elle est porteuse de distinction entre ce qui est conforme à la norme et qui mérite la protection projetée d'une part, et ce qui ne satisfait pas aux conditions prévues par la norme et ne peut donc être éligible au chapitre de la protection de la loi, d'autre part. Il faut se représenter toute situation qui, lorsqu'elle remplirait certaines conditions exigées par la loi, pourrait revêtir la qualification de situation de droit. Une opposition a ainsi toujours été entretenue entre situations de droit et situations de fait 6 . Il est alors aisément compréhensible que ces dernières renvoient à un ensemble de situations juridiques irrégulières, dégradées, dégénérées, constituant des doublets de situations régulières, mais ne remplissant en général pas leurs conditions de formalisme ${ }^{7}$. La réaction du droit ne s'est pas souvent limitée à leur simple qualification en situation de fait.

Une démarche sanctionnatrice caractérise justement cette réaction à l'égard des situations de fait qui sont privées d'effets et donc du bénéfice de la protection prévue pour la situation juridique correspondante. Malgré cette position du droit, l'existence des situations de fait semble s'imposer. Celles-ci tendent à envahir toutes les sphères du droit, se développant soit de manière originelle ${ }^{8}$, soit de manière dérivée ${ }^{9}$. Le droit des

l'illusoire limpidité du droit ", (1999) 41 Revue Droit et société 85, 86; Théodore IVAINER, L'interprétation des faits en droit, Paris, LGDJ, 1988.

5. Voir Roger Houin, "Extrait du rapport général aux Journées de Lille de l'Association Henri Capitant ", (1958) 10 RIDC 82, 83.

6. Voir Laurent LEVEnEUR, Situations de fait et droit privé, Paris, LGDJ, 1990; R. HouIN, préc., note 5, 82

7. Voir Jean CARBOnNIER, Flexible droit, pour une sociologie du droit sans rigueur, 10e éd., Paris, LGDJ, p. 35; R. HouIN, préc., note 5, 84.

8. C'est le cas d'un associé commanditaire qui, n'ayant justement pas qualité, choisit de poser des actes de gestion. C'est également le cas des 
affaires, en général, en recèle un grand nombre. La notion de commerçant de fait y est bien connue ${ }^{10}$ comme caractérisant la situation d'une personne qui exerce les actes de commerce sans requérir son immatriculation au registre du commerce et du crédit mobilier ${ }^{11,12}$. Une telle personne s'est mise en marge de la législation et pose le problème du régime des actes qu'elle a passés à l'égard des tiers. En règle générale, le droit le prive du bénéfice desdits actes tout en permettant aux tiers de s'en prévaloir contre lui ${ }^{13}$. Cette solution trouve sa raison d'être en droit des sociétés.

La législation des sociétés connaît particulièrement l'existence de nombreuses situations de fait à côté des situations de droit que sont la société commerciale et le dirigeant social. Pourtant, le droit des sociétés a un caractère poussé d'ordre public ${ }^{14}$. Malgré cela, l'on doit se rendre à l'évidence des institutions comme la société de fait, le dirigeant de fait, la société

personnes qui choisissent de créer une société soumise à la formalité de l'immatriculation et qui s'abstiennent de le faire.

9. La situation de fait est dérivée lorsqu'elle découle d'une dégénérescence de situation de droit. C'est le cas de la société devenue de fait par suite de son annulation par le juge (Combinaison des articles 201 et 253 de l'Audsc et Gie).

10. Voir Guité Diop, "Le commerçant de fait ", mémoire de maîtrise, Dakar, Université Cheikh Anta Diop, 2007.

11. Il ressort de la lecture combinée des articles 34 et 36 de l'Acte uniforme de l'OHADA, relatif au droit commercial général (Audcg, tel que révisé le 15 décembre 2010), que le registre du commerce et du crédit mobilier (RCCM) est un système, composé des fichiers nationaux et d'un fichier régional, destiné à permettre l'immatriculation des professionnels et l'information des tiers. Yvette-Rachel KALIEU ELONGO, "Le rôle du registre du commerce et du crédit mobilier OHADA dans l'amélioration de l'accès au crédit ", dans A. AKAM AKAM (dir), Les mutations juridiques dans le système OHADA, Paris, l'Harmattan, 2009, p. 133.

12. L'immatriculation au RCCM est une obligation professionnelle pour tout commerçant, ainsi que pour l'entreprenant nouvel exploitant de l'activité commerciale introduit par la révision du 15 décembre 2010. Cette obligation est exprimée aux articles 44 et 46 de l'Audcg.

13. Art. 61 Audcg.

14. Pascal NGuiHe KAnTE, "Le caractère d'ordre public du droit uniforme des sociétés commerciales en Afrique ", dans A. AKAM AKAM (dir), Les mutations juridiques dans le système OHADA, Paris, l'Harmattan, 2009, p. 181 . 
créée de fait, le groupe de sociétés ${ }^{15}$, etc. Ces institutions sont même légalement aménagées avec parfois un régime bien construit. Il est, par exemple, prévu pour la société de fait, qu'elle soit soumise au régime de la société en nom collectif ${ }^{16}$, comme la société en participation. Pourtant, les deux entités constituent des sociétés sans personnalité morale. L'on s'attendrait normalement, dans le cas par exemple de la société créée de fait, que les acteurs en soient privés du bénéfice des effets de la constitution d'une société. Or, l'article 866 de l'Acte uniforme de l'OHADA, relatif au droit des sociétés commerciales et du groupement d'intérêt économique, donne la possibilité à quiconque y ayant intérêt de faire la preuve de l'existence d'une société de fait entre elle et d'autres, afin de pouvoir en obtenir la liquidation ${ }^{17}$. Le régime de la société en nom collectif qui est ici appliqué est reconnu pour être des plus sévères. On pourrait donc légitimement créditer le législateur d'un souci de protection des parties faibles. Il ne serait pas a contrario exagéré de lui objecter une certaine complaisance à l'égard des parties devant leur refus d'accomplir les formalités exigées afin de se mettre sous la protection de la loi. En tout état de cause, la démarche décrite ci-dessus est particulière au droit des sociétés.

À l'observation donc, la position de cette législation se situe au carrefour de deux approches alternatives qui caractérisent souvent les pouvoirs publics dans le traitement des situations de fait : soit la loi ou le juge évince la situation de fait pour protéger la situation juridique correspondante, tel est le cas du regard

15. Le groupe de sociétés, par exemple, a toujours été considéré comme une situation de fait à cause de son défaut de personnalité morale. Voir Jean GATsi, "Filiale, succursale, groupe de sociétés", dans P.-G. Pougoue (Dir.), Encyclopédie du droit OHADA, Lamy, 2011, p. 793; Mayatta Ndiaye MBAYE, "Les groupes de sociétés dans l'OHADA ", (2004) 848 Penant, 265; Mamadou Kone, "La notion de groupe de sociétés en droit OHADA ", (2006) Penant 285; Yves GuYON, Droit commercial et des sociétés, 9e éd., Paris, Économica, 1996, n 613, p. 639 et suiv; Bruno OpPETiT et Alain SAYAG, "Méthodologie d’un droit des groupes de société ", (1973) Revue des sociétés 577.

16. Art. 868 Audsc et Gie.

17. Infra, p. 18. 
porté dans notre pays, au concubinage, à l'enfance naturelle ${ }^{18}$, etc.; soit ils admettent la situation de fait en marge de la situation de droit tout en pondérant les effets de la première. C'est donc cette dernière approche qui semble avoir prévalu en droit des sociétés.

La présente étude vise à comprendre cette position du droit par rapport aux situations de fait sur le point de savoir si la réceptivité du droit des sociétés à l'égard des situations de fait, notamment la prolifération de ces entités au sein de son champ d'application, affecte-t-elle son efficacité ou alors en est-elle un élément de renforcement? Quelle est la place des situations de fait dans le droit des sociétés? En d'autres termes, quel est le sort qui leur est réservé par le droit des sociétés? La problématique ainsi posée a un intérêt certain dans la mesure où elle vise à justifier le choix du législateur des sociétés et du juge quant au traitement réservé aux situations de fait.

À tout prendre, la position du droit des sociétés paraît logique. Elle participe vraisemblablement d'une certaine souplesse indispensable dans le domaine des affaires. En effet, une indifférence à l'égard des situations de fait conduirait probablement à l'exacerbation des difficultés d'une entreprise si, par exemple, le dirigeant de fait qui a posé des actes ruineux ne peut pas être condamné au même titre qu'un dirigeant de droit. L'insécurité caractériserait la situation des tiers s'ils ne pouvaient obtenir la condamnation, à leur profit, des personnes qu'ils ont cru constituer une société et avec lesquelles ils ont été en relations d'affaires; ou si la nullité d'une société avec laquelle ils ont contracté devait produire des effets rétroactifs du régime classique des nullités.

18. Sous le droit camerounais, par exemple, la concubine délaissée n'a aucun recours contre son amant, sa situation étant non reconnue par le droit de la famille. 
Le droit sait en effet corriger sa rigueur pour s'adapter progressivement aux faits ${ }^{19}$. Comme le soulignait un auteur, il est fort "salutaire que le droit ne soit pas cette massue, ce sceptre qu'on voudrait qu'il fût "20. C'est certainement cette logique qui sous-tend le législateur des sociétés dans l'accueil qu'il réserve aux situations de fait $(\S \mathrm{I})$, contrairement aux autres disciplines du droit. Il faut dire que cette démarche n'est pas complaisante à l'égard des situations de fait, elle vise l'efficacité du système, la prise en compte des situations de fait contribuant à l'essor du droit des sociétés (§II).

\section{§ I- L'accueil des situations de fait par le droit des sociétés}

Le postulat que le droit des sociétés accueille les situations de fait doit être compris dans le sens de l'aménagement qu'il leur apporte en termes de régime juridique. Il ne s'agit pas d'un jugement de valeur contre les autres législations, les situations de fait demeurant des cas de méconnaissance du formalisme de certaines institutions juridiques. La démarche du droit des sociétés en recourant à cette technique est, d'une part, d'encadrer un maximum de situations dans le circuit de l'économie afin de réduire l'informel et, d'autre part, de traquer les auteurs de la violation des normes afin de protéger efficacement les parties faibles. Pour cela, il faut reconnaître que les situations de fait constituent en droit des sociétés de véritables catégories, peut-être à part, que l'on peut étudier sous deux grands ensembles à savoir, d'une part, les groupements d'affaires sans personnalité morale (A) et, d'autre part, l'administration des sociétés sans mandat (B).

\section{A- Les groupements d'affaires sans personnalité morale}

Les groupements d'affaires sans personnalité morale sont une réalité en droit des sociétés ${ }^{21}$ alors que cet attribut est, en

19. Contra, Christian ATIAs, "Le mythe de l'adaptation du droit au fait ", (1977) Revue Dalloz, 251.

20. Jean CARBONNIER, préc., note 7, p. 379.

21. Bérenger Yves MEUKE, "La société non personnalisée dans l'OHADA. Étude de l'impact de l'absence de personnalité morale dans la société en 
droit OHADA, le stade de complétude de la fiction juridique qui caractérise les sociétés ${ }^{22}$. Leur existence constitue une entorse à la théorie actuelle de la personnalité morale ${ }^{23}$ et en fait des situations de fait à côté des catégories normales dotées de la personnalité morale. Cette réalité se vérifie à propos de la société de fait (1) et d'autres groupements sans personnalité morale (2).

\section{1- La société de fait}

La société de fait est l'une des situations à propos desquelles le législateur des sociétés commerciales s'est toujours expressément illustré. Le droit OHADA, en particulier ${ }^{24}$, permet de l'entendre comme celle qui existe en marge des formalités légales prescrites à cet effet. Évidemment, ce postulat contraste d'apparence avec les définitions telles que proposées par le législateur. Un auteur y voit même avec insistance une confusion des deux notions classiques de société de fait et société créée de fait25. Mais cet état de choses est sans influence sur la

participation ", (2006) 12 Revue juridique tchadienne 6; Claude BouTRY, "L'absence de personnalité morale dans les sociétés ", (2000) 51/52 Semaine Juridique 1855; éd. E, 2001, n 7/8, p. 310.

22. Cette conception de la société n'est certainement pas universelle. Au Québec par exemple, le Code civil semble dénier la personnalité juridique à plusieurs types de sociétés à l'instar de la société en nom collectif, en commandite ou en participation. Seule bénéficie de la personnalité morale la société par actions encore appelée compagnie (art.2188 C.c.Q). Voir, Donald A. RiEndEAU, "La "société" en droit québécois ", (2003) 63 Revue du Barreau 127.

23. Sous la législation des sociétés de l'OHADA, la personnalité morale constitue une disposition légale. Elle ne tombe plus de tout sens comme la jurisprudence l'a voulu en son temps. L'article 98 de l'Audsc et Gie dispose d'ailleurs que "[t]oute société jouit de la personnalité juridique à compter de son immatriculation au RCCM, à moins que (l'Acte uniforme) n'en dispose autrement ". Voir, Denis PoHe ToPKA, "Personnalité morale des sociétés ", dans P.-G. Pougoue (dir.), Encyclopédie du droit OHADA, Lamy, 2011, p. 1344; voir également Jean PAILlusseau, "Le droit moderne de la personnalité morale ", (1993) RTD. civ. 705;

24. Les articles 864 et suivants de l'Acte uniforme relatif au droit des sociétés commerciales et du groupement d'intérêt économique.

25. Voir, Charles MBA-OwONO, "Société de fait et société créée de fait : une distinction empreinte de confusion en droit uniforme OHADA ", dans X. 
catégorisation de la société de fait en situation de fait d'autant plus que le législateur semble simplement entériner la volonté des parties qui se mettent en marge des règles impératives (a), même si c'est au juge qu'il reviendra en définitive de déterminer s'il y a ou non société de fait (b).

\section{a- La prise en compte de la volonté des associés de fait}

La lecture des dispositions de l'Acte uniforme relatif au droit des sociétés révèle justement que le législateur prend en compte la volonté des associés de fait de créer une société de fait. Cela résulte très facilement, dans deux séries de dispositions, de la nomination de cette situation à laquelle un régime est construit.

Ainsi, selon l'article 864 : "Il y a société de fait lorsque deux ou plusieurs personnes physiques ou morales se comportent comme des associés sans avoir constitué entre elles l'une des sociétés reconnues par (1'Acte uniforme) "26. Il y a également société de fait aux termes de l'article 865 "[1]orsque deux ou plusieurs personnes physiques ou morales ont constitué entre elles une société reconnue par (l'Acte uniforme), mais n'ont pas accompli les formalités légales constitutives ou ont constitué entre elles une société non reconnue par (l'Acte uniforme)". Ces hypothèses laissent assurément entrevoir la société créée de fait qui existe " [s]i contrairement aux dispositions (de l'Acte uniforme), le contrat de société ou, le cas échéant, l'Acte unilatéral de volonté n'est pas établi par écrit et que, de ce fait, la société ne peut être immatriculée "27. En effet, le dénominateur commun entre les deux catégories de sociétés, telles que prévues à l'Acte uniforme,

HENRY (dir), Des contrats civils et commerciaux aux contrats de consommation, Mélanges en l'honneur du Doyen Bernard Gross, Presses Universitaires de Nancy, 2009, p. 467 et suiv.; Ohadata D-10-19; "Sociétés de fait ", dans P. G. Pougoue (dir) Encyclopédie du droit OHADA, éd. Lamy, 2011, p. 1958-1967.

26. TGI Ouagadougou (Burina Faso), Jugement commercial $\mathrm{n}^{\circ} 215 \mathrm{du} 21$ février 2001 : STTP Sarl c/ Société africaine de services SA et SOFITEX, dans Jean GATSI, OHADA, Code des sociétés commenté et annoté, Douala, PUL, 2011, note sous art. 864 de l'Audsc et Gie, p. 186.

27. Art. 115 de l'Audsc et Gie. 
est l'existence de la société en marge des formalités légales édictées pour la constitution des sociétés commerciales de l'OHADA. C'est ce qui entraîne d'ailleurs leur absence de personnalité morale. Il est difficilement pensable que l'absence de contrat de société écrit ne laisse pas déduire l'existence de la société du comportement des associés ou que le défaut d'accomplissement des formalités légales même en présence d'une société reconnue par l'Acte uniforme ne s'assimile pas à la société créée de fait dès lors qu'elle n'aura pas la personnalité morale pour défaut d'immatriculation. Sur cette base, la distinction n'est pas très nette entre la société de fait proprement dite et la société dite créée de fait ${ }^{28}$. D'autant plus que le législateur OHADA semble avoir adopté le modèle de société de fait, au moins au plan terminologique, tel qu'il existe en droit français. À propos de ce système juridique justement, il est reconnu que la distinction entre société de fait et société créée de fait est définitivement clarifiée.

La société de fait est communément reconnue comme une société de droit dégénérée, issue de l'annulation d'une société grevée d'une cause de nullité, mais bénéficiant du privilège de non-rétroactivité du régime de nullité des sociétés ${ }^{29}$. C'est certainement l'hypothèse prévue à l'article 253 de l'Acte uniforme qui dispose : "Lorsque la nullité de la société est prononcée, elle met fin, sans rétroactivité, à l'exécution du contrat. Il est procédé à sa dissolution et, pour ce qui concerne les sociétés pluripersonnelles, à leur liquidation ". En règle générale, la personnalité de la société subsiste lors de la dissolution, pour les besoins de la liquidation ${ }^{30}$.

28. François-Xavier LuCAS, "La société dite "créée de fait" ", dans Mélanges offerts à Guyon, Dalloz, 2003, p. 738.

29. Art. 253 de l'Audsc et Gie sur la nullité des sociétés. Voir, Kassia Bi OulA, "Le recul de la nullité dans l'Acte uniforme sur les sociétés commerciales et le GIE ", (2004) 848 Penant 252; R. GuilliEN et J. VincEnT, préc., note 3, p. 611; Rep. Soc. Dalloz, 1971, V Société de fait, $\mathrm{n}^{\circ} 1$; Joseph HEMART, Traité des nullités de sociétés et des sociétés de fait, Paris, Sirey, 1926.

30. Art. 201 al. 3 de l'Audsc et Gie. 
La distinction entre société de fait et société créée de fait ne résiderait qu'à l'égard de leurs processus de création respectifs. À ce titre, la société créée de fait peut être considérée comme un avorton du droit des sociétés. Elle n'a donc jamais pu atteindre le stade normal de l'espèce à laquelle elle appartient ${ }^{31}$; alors que la société de fait a atteint ce stade, a même fonctionné avant d'en avoir été déchue par l'annulation. C'est ce qui fait qu'elle existe dans les faits pour éviter l'effet de la rétroactivité et permettre la liquidation des opérations restant en cours. Tout au plus pourraiton admettre que le législateur OHADA traite de la société créée de fait comme d'un cas de société de fait, ce qui est fondé en théorie du droit.

Dans le sens de cette théorie générale du droit, la société de fait appartient bien à la catégorie des situations de fait, et la distinction sus-évoquée n'y change rien. Il s'agit bien d'une société sans personnalité morale ${ }^{32}$ qui ne peut donc être qualifiée de société de droit. C'est cette caractéristique des sociétés de fait et société créée de fait qui nous intéresse ici au regard du sort que leur réserve finalement le droit des sociétés. En complément de la construction légale ci-dessus, c'est au juge de déterminer s'il y a ou non société de fait.

\section{b- L'office décisif du juge dans la caractérisation de la société de fait}

Quelle que soit la place faite à la volonté des parties dans la création d'une société de fait, c'est au juge qu'il appartient de dire s'il y a ou non société de fait. En prévoyant qu'il puisse être reconnu l'existence d'une société entre des personnes physiques ou morales qui se comportent comme des associés sans avoir constitué entre elles l'une des sociétés reconnues par l'Acte

31. Il s'agit de l'espèce des sociétés en général.

32. L'article 115 de l'Audsc et Gie dispose pour la société créée de fait qu'elle n'a pas la personnalité juridique. Même la société devenue de fait par application de l'article 253 de l'Audsc et Gie ne dispose de la personnalité morale que pour les besoins de sa liquidation (201, al. 3). 
uniforme relatif au droit des sociétés ${ }^{33}$, le législateur s'adresse assurément au juge. Il en va de même lorsque deux ou plusieurs personnes physiques ou morales auront constitué entre elles une société reconnue par l'Acte uniforme, mais n'ont pas accompli les formalités légales constitutives ou ont constitué entre elles une société non reconnue par l'Acte uniforme ${ }^{34}$.

L'article 868 de l'Acte uniforme relatif au droit des sociétés commerciales et du groupement d'intérêt économique est d'ailleurs fort éloquent à ce propos lorsqu'il précise que " [1]orsque l'existence d'une société de fait est reconnue par le juge, les règles de la société en nom collectif sont applicables aux associés ".

La reconnaissance de la société de fait intervient à titre de sanction, notamment pour les besoins de la liquidation entre associés ou pour le désintéressement des créanciers. En réalité, les associés ont cru créer entre eux une société de droit en ignorant le vice qui entache leur structure. L'annulation pour ce vice doit laisser subsister la société à laquelle seront appliquées les règles dévolues aux sociétés de droit. Le droit des sociétés impose donc aux associés l'existence de leur société dans les faits. De l'autre côté, les associés ignorent leur appartenance à une société, mais les actes qu'ils posent en tout cas, à l'égard des tiers, les y maintiennent. Lorsque le juge est saisi d'une demande de reconnaissance de l'existence d'une société de fait, il pourvoit ainsi que l'y habilite la loi.

Cependant, les définitions, telles que suggérées par le législateur, ne rendent pas totalement compte du contenu de la notion de société de fait. En effet, un certain nombre de conditions sont nécessaires pour cette reconnaissance. Il s'agit des conditions de validité d'une société à savoir les apports, la volonté commune d'exploitation et la volonté de partager les bénéfices ainsi que de

33. Art. 864 de l'Audsc et Gie. Même la société devenue de fait par application de l'article 201, al. 3 de l'Audsc et Gie.

34. Art. 865 de l'Audsc et Gie. 
contribuer aux pertes ${ }^{35}$, qu'il appartient au juge de vérifier afin de juger s'il y a ou non société de fait. En dehors des cas évoqués cidessus, certains autres groupements constituent des situations de fait.

\section{2- Les autres groupements d'affaires sans personnalité morale}

Il n'y a pas que la société de fait qui puisse être étudiée dans la catégorie de groupement d'affaires sans personnalité morale et donc des situations de fait. La législation des sociétés vise également la société en participation (a) au chapitre consacré à la société non immatriculée. Ce dernier concept nous permet d'étudier la société avant son immatriculation comme une situation de fait (b).

\section{a- La société en participation}

La société en participation est celle dans laquelle les associés conviennent qu'elle ne sera pas immatriculée au registre du commerce et du crédit mobilier et qu'elle n'aura pas la personnalité morale ${ }^{36}$. Cette définition est celle fournie par l'Acte uniforme relatif au droit des sociétés commerciales et du groupement d'intérêt économique. De manière expresse donc, la société en participation est dispensée de la formalité de l'immatriculation. Quand on sait l'effet que produit cette formalité

35. Art. 4 de 1'Audsc et Gie : CA Ouagadougou, Arrêt n 86 du 21/04/2006, Affaire: Adoko Sessinou Bernard c/ NAcoulma Désiré, Yanogo B. Michael, Songnaba / Compaore Claudine, www.ohada.com; CA Daloa, 1 ère Civ \& Com., n 195/2000 du 24 Mai 2000 : WAN Kil LeE c/ JEON KuK Hyun, dans J. GATSI, OHADA, Code des sociétés commerciales et du GIE commenté et annoté, préc., note 15, p. 187; Bakary DiALLO, "La société créée de fait dans l'OHADA ", note sous CCJA n 31, 04 novembre 2004 : Ayant droit de B. c/ Madame A, art. précité, p. 18; À titre de droit comparé Marie-Hélène MALEVILLE, "Sociétés et groupement particuliers Société créée de fait ", note sous Cass. com., 5 avril 2005, en ligne: $<$ www.institut-idef.org $>$.

36. Art. 854 Audsc et Gie. Voir Antoine DELABRIERE, "La société en participation dans l'OHADA ", (2004) 848 Penant 396; B.-Y. MEukE, préc., note 21, p. 6. 
sur l'existence de la société, il est aisé de conclure que la société en participation est une situation de fait. La raison d'un tel postulat découle d'une comparaison avec les sociétés de droit et la société de fait évoquée plus haut.

Comparée à la société de fait qui correspond à la catégorie de situation de fait, la société en participation contient tous les éléments d'assimilation à cette dernière ${ }^{37}$. Elle est une société voulue selon l'une des formes de sociétés prévues à l'Acte uniforme $^{38}$. Il est permis de penser ainsi du moment qu'elle est régie dans le même corps de règles qui organisent les sociétés en nom collectif, à responsabilité limitée, en commandite simple et anonyme. Sauf à présumer les futurs associés en participation titulaires de l'érudition nécessaire pour imaginer une forme particulière d'organisation qu'ils soumettront au régime de la société en participation, ceux-ci auront simplement à l'esprit les modèles de sociétés que leur fournit la législation en vigueur. Or, ces sociétés ont été bien aménagées en termes de conditions de forme et de fond. Seulement, la société en participation doit évoluer en marge de ces conditions. Elle n'a donc pas la personnalité morale; elle n'est pas davantage soumise à publicité. Par voie de conséquence, elle n'a pas d'existence ostensible, seules les parties en ont connaissance. De plus, à l'égard des tiers, chaque associé contracte en son nom personnel et est seul responsable, mais comme dans la société de fait, par application de la théorie de l'apparence ${ }^{39}$, les tiers peuvent engager la responsabilité indéfinie et solidaire des associés qui ont agi ès qualités.

37. Par exemple, Jocelyne VALlansan et Éric DeSMORIEUX, Société en participation et société créée de fait: aspects juridiques et fiscaux, Coll. "Pratique des affaires ", Paris, GLN-Joly, 1996.

38. Art. 6, al. 2 : La société en nom collectif (SNC), la société à responsabilité limitée SARL, la société en commandite simple (SCS) et la société anonyme (SA).

39. Selon l'alinéa 2 de l'article 854, l'existence de la société en participation est prouvée par tout moyen. 
Comparée aux sociétés de droit, la société en participation ne peut pas être assimilée à ces dernières dès le moment qu'elle est introduite par le législateur comme une société constituant une exception par rapport à la forme rigoureuse des quatre sociétés prévues dans l'Acte uniforme sur les sociétés et le groupement d'intérêt économique. Le législateur indique bien pour l'instituer que " [p]ar exception aux dispositions qui précèdent, les associés peuvent convenir que la société ne sera pas immatriculée "40. Les dispositions visées ici, faut-il le rappeler, imposent à toute société de droit qu'elle soit immatriculée au registre du commerce et du crédit mobilier et font de cette formalité la condition d'acquisition de la personnalité morale ${ }^{41}$. Plus encore, elles sont d'ordre public ainsi que le prévoit l'article 2 de l'Acte uniforme ${ }^{42}$.

La formulation des articles 114 et 854 de l'Acte uniforme sur les sociétés induit que les associés peuvent déroger par leurs conventions particulières aux dispositions d'ordre public du droit des sociétés. Cela ne pose pas de problème de cohérence du système, car la loi qui l'interdit peut encore l'autoriser. Mais la qualification, la catégorisation de ce type de société n'ont pas été réglées. La position qui paraît donc évidente, au vu des développements qui précèdent, est celle selon laquelle la société en participation est une situation de fait. Ce d'autant plus que, même résultant d'une habilitation à méconnaître le formalisme des sociétés, elle ne constitue pas une organisation rigoureusement particulière. Il s'agit d'une société voulue selon l'un des modèles prévus par l'Acte uniforme ${ }^{43}$, mais qui ne jouira pas de la personnalité morale du fait du défaut d'immatriculation. Cette conséquence la déchoit de la catégorie des sociétés de droit, quelle que soit l'alchimie concevable, pour la maintenir dans celle des situations de fait. C'est la même déduction qui est faite à propos de la société avant son immatriculation.

40. Art. 114 Audsc et Gie.

41. Art. 97 et 98 Audsc et Gie. Voir, D. PoHE TopkA, préc., note 23, p. 1344.

42. Voir, P. NGUIHE KANTE, préc., note 14, p. 181.

43. Rappelons que selon l'article 856 de l'Acte uniforme son régime, par défaut, est celui de la société en nom collectif. 


\section{b- La société avant son immatriculation}

Comme nous l'avons rappelé en amont, la société acquiert le statut de situation de droit dès lors qu'elle est immatriculée au registre du commerce et $\mathrm{du}$ crédit mobilier ${ }^{44}$. Cela a pour conséquence qu'elle acquiert la personnalité morale. Or la vie de la société ne commence pas que dès l'acquisition de la personnalité morale, cette dernière formalité ne devant être regardée que comme le critère de sa complétude ${ }^{45}$. Deux stades de la société et non des moindres lui permettent de poser les jalons de son existence et qui constituent de véritables situations de fait. Il s'agit de la société en formation et de la société constituée, mais non encore immatriculée.

La société en formation est définie par le Nouveau Dictionnaire Juridique comme étant la société non encore constituée $^{46}$. Cette définition, conforme à l'article 100 de l'Acte uniforme relatif au droit des sociétés commerciales et du groupement d'intérêt économique, cache mal toute la dynamique qui caractérise la société en formation. Ne l'entrevoir que sous l'angle de sa différence d'avec la société constituée, c'est vouloir lui nier toute existence. Or la société en formation existe, vit et prend des engagements, au moins par l'intermédiaire des fondateurs, qui sont susceptibles d'engager la future société devenue situation de droit à la suite de l'immatriculation.

Du fait donc que les actes pris lors de la société en formation soient susceptibles d'être repris avec effet rétroactif au jour de la société en formation, ne peut que conforter la thèse de l'existence (latente si l'on veut) de la société. En tout état de cause, le droit des sociétés n'est pas indifférent à la réalité que constitue la société en formation, comme semble l'être le droit civil à l'égard des fiançailles qui précèdent le mariage civil.

44. Art. 98 et 29 de l'Ausdc et Gie; D. PoHE TopkA, préc., note 23 , p. 1344.

45. Ce point de vue doit même être tempéré dès le moment que le législateur a habilité la société en participation à fonctionner sans personnalité morale

46. J. Gatsi, Nouveau Dictionnaire Juridique, préc., note 2, p. 295. 
L'on doit seulement s'intéresser à sa catégorisation entre situation de fait et de droit. Il est alors évident que la société en formation est une situation de fait, même s'il faut lui reconnaître une existence sur le plan économique au moins.

S'agissant de la société constituée, il est vrai qu'elle est le stade où la société est véritablement mise sur pied, à travers la signature des statuts, la nomination des organes dirigeants, leur prise de fonction, mais cela ne change pas grand-chose à sa nature juridique. En effet, justement, la société constituée, mais non encore immatriculée ne devient une situation véritable de droit qu'à partir de son immatriculation ${ }^{47}$. C'est à partir de cet instant qu'elle peut jouer son rôle public, qu'elle peut agir envers les tiers. S'agissant de son existence juridique, c'est avec l'immatriculation que la société est véritablement consolidée. La société est soumise à une sorte de condition résolutoire de l'immatriculation dans un délai de six mois à compter du premier dépôt des fonds chez un notaire ou dans un compte à cet effet. Il s'en suit que si la société n'a été immatriculée chaque souscripteur peut se faire autoriser, par la juridiction compétente, à retirer ses fonds. Sachant que la société ne peut exister sans capital social et que l'immatriculation est elle-même conditionnée par l'existence d'un capital souscrit et libéré au moins pour fraction ${ }^{48}$, elle existe depuis avant cette immatriculation, mais dans les faits. Il s'en suit donc que la société constituée, mais non encore immatriculée est une situation de fait.

\section{B- L'administration des sociétés sans mandat}

Le système d'administration des sociétés prévu dans l'Acte uniforme relatif au droit des sociétés et du groupement d'intérêt

47. Michel GERMAIN, "Naissance et mort des sociétés commerciales ", dans X. BlANC-JOUVAN (dir), Aspects actuels du droit commercial français. Études dédiées à René Roblot, Paris, LGDJ, 1984, p. 217.

48. La précision est donnée à ce sujet, pour la société anonyme, à l'article 388 de l'Acte uniforme : CA Abidjan, $n^{\circ} 1060$ / 2000 du 1er Décembre 2000, K. c/ Z et T, Le Juris-Ohada, $\mathrm{n}^{\circ}$ 3/2003, Juillet-Septembre 2003, p. 43. 
économique, à l'instar de la grande majorité des législations sur les sociétés, est fondé sur le mandat du dirigeant social. Celui-ci ne pose des actes que pourvu qu'il ait reçu pouvoir soit des associés en situation normale, soit du juge en situation de crise. En dehors de ces deux cas, toute immixtion dans la gestion d'une société est interdite. Mais il arrive qu'en violation de cet interdit, une personne, associee ou non, participe de manière significative à la gestion de la société. Le droit des sociétés a qualifié cette situation de direction de fait qui est caractérisée aussi bien au regard de la personne du dirigeant de fait (1) que de ses actes (2).

\section{1- Les critères de la direction de fait tenant à la personne}

Le dirigeant de fait est toute personne qui, sans avoir reçu un mandat social ou, alors que le mandat qui lui a été donné était nul ou n'était plus valable, s'est immiscé dans la gestion d'une société $^{49}$. De la définition, l'on déduit que le dirigeant de fait peut être soit un ancien dirigeant dont le mandat n'est plus valable, soit une personne n'étant aucunement pressentie à la direction d'une société, mais qui pose des actes de gestion. Son existence est le signe véritable d'une méconnaissance des règles d'ordre public concernant le système d'administration des sociétés.

Le cas de l'ancien dirigeant de droit qui devient de fait peut être compréhensible du moment qu'ayant été aux affaires, il garde quelques liens avec la société. Il peut lui être plus facile d'influer sur la direction de la société, soit de façon directe, soit de façon indirecte. Dans la plupart des cas d'ailleurs, le dirigeant de droit peut être un associé50. Il demeure donc dans la société même

49. Jean-Louis Rives-LANGE, "La notion de dirigeant de fait (au sens de l'art. 99 de la loi du 13 juill. 1967 sur le règlement judiciaire et la liquidation des biens) ", (1975) 5 Revue Dalloz 41; Jean-Marie MACQuERON, "La notion de dirigeant de fait : étude de la jurisprudence française de ses origines à 1981 ", thèse de doctorat Rouen, Université de Rouen, 1982; Daniel TRICOT, "Les critères de la gestion de fait ", (1996) Droit et patrimoine 24; Serge BRAUDO, Dictionnaire de droit privé, en ligne: $<w w w . d i c t i o n n a i r e-j u r i d i q u e . c o m>$ (consulté le 30 décembre 2011).

50. Art. 276 (SNC), 298 (SCS) et 323 (SARL). Cette faculté n'est pas exclue pour les sociétés anonymes. 
ayant perdu son mandat. Malgré tout, rien ne justifie sa gestion de fait, pas plus n'y a droit la personne qui n'a jamais été pressentie à la fonction d'administrateur. Le cas de cette dernière personne désigne la situation soit d'un tiers à la société, soit même d'un associé, qui sans avoir de rapports avec l'administration de la société, s'immisce dans la gestion. Le phénomène de l'administration des sociétés sans mandat constitue donc une réalité en droit des sociétés.

Curieusement, il n'en existe pas de grande évocation dans l'Acte uniforme relatif au droit des sociétés commerciales et du groupement d'intérêt économique. Tout au plus pourrait-on le déduire des dispositions de l'article 300 (à propos de la société en commandite simple) de l'Acte uniforme qui étendent la responsabilité d'un dirigeant à un associé commanditaire qui aurait posé un acte de gestion ${ }^{51}$. Évidemment, cette extension ne suffit pas pour rendre compte de la notion de dirigeant de fait. Il faut se tourner vers l'Acte uniforme portant organisation des procédures collectives d'apurement du passif52 et surtout de la jurisprudence pour comprendre la notion de dirigeant de fait existant en droit des sociétés.

À l'origine, c'est justement à la jurisprudence que l'on doit d'avoir systématisé la notion de dirigeant de fait ${ }^{53}$. Il faut alors s'y référer pour en avoir une vue complète que possible.

Ainsi, lorsque des actionnaires réussissent à mettre le conseil d'administration dans une telle situation de dépendance que ses décisions s'avèrent soumises aux résultats de leurs recherches et leurs avis, l'on doit retenir la qualité de dirigeant fait contre eux. De même, une personne morale tierce à la société peut avoir la qualité de dirigeant de fait, pour avoir en fait et par l'intermédiaire

51. André AKAM AKAM, "La responsabilité civile des dirigeants sociaux en droit Ohada ", (2007) 21 (2) Revue internationales de droit économique 234.

52. Ce corps de règles sera examiné dans la deuxième partie de ce travail.

53. Voir, Jean GATSI, "La jurisprudence, source du droit OHADA ", (2012) 64 (2) Revue internationale de droit comparé 477; www.juriscope.org, 2012. 
d'un administrateur dépourvu de toute autonomie à son égard, réalisé en toute indépendance, des actes positifs de gestion. Tel est le cas d'une société holding à l'égard de laquelle la qualité de dirigeant de fait des filiales de la société mère, avait été reconnue, après qu'est été mise en évidence l'absence totale d'autonomie de ces filiales à l'égard du holding "54.

\section{2- Les critères de la direction de fait tenant aux actes}

Pour qu'une personne soit qualifiée de dirigeant de fait, il faut qu'elle ait accompli des actes positifs de gestion. Par acte positif de gestion, il faut entendre un acte soit de gestion courante, soit d'administration, soit conservatoire ou de disposition. L'acte doit donc être accompli en situation d'immixtion, c'est-à-dire à la place ou aux côtés des personnes normalement autorisées à l'accomplir. En effet, pour une raison ou une autre, une personne n'en ayant pas qualité peut participer à la prise des décisions. C'est généralement dans le but de faire passer ses intérêts. Tel un banquier qui impose à son client l'orientation de l'activité en raison de la relation de crédit dont l'autre est dépendant ${ }^{55}$.

L'acte doit être accompli de manière continue telle que la personne soit réputée avoir exercé le contrôle effectif et constant de la marche de la société. Le propre de la direction de fait, c'est justement la prise de contrôle informelle de la marche de l'entreprise. Cette prise de contrôle se fait soit de manière directe, par prise de décision ou participation à des décisions, soit de manière indirecte par l'exercice d'une pression sur les décideurs. C'est le cas souvent d'un associé majoritaire ou du banquier dispensateur de crédit. Il est vrai que les juges se montrent particulièrement prudents quant à la qualification d'une banque, de dirigeant de fait. Ils recherchent l'étroitesse des relations caractérisée, par exemple, par l'existence d'un pacte d'actionnaires

54. Pour l'ensemble de ces questions, lire J. GATSI, préc., note 53.

55. Monique Aimé Mouthieu, L'intérêt social en droit des sociétés, Paris, l'Harmattan, 2009, p. 141; Anne Laure CAPOEN, "La responsabilité du banquier à l'égard des entreprises en difficultés ", thèse de doctorat Toulouse, Université de Toulouses, 2008, p. 299 et suiv. 
entre une société et la banque, ayant une participation financière dans la première, au terme duquel cette dernière aurait le droit de nommer au moins un administrateur dans la société. Par un tel pacte, une banque a pu nommer deux de ses cadres dans l'entreprise, c'est tout naturel lors du traitement des difficultés de l'entreprise, elle fut déclarée dirigeante de fait, en raison justement du lien de subordination entre elle et les administrateurs désignés et la faute de gestion qu'ils ont commise ${ }^{56}$.

Dans la pratique, le dirigeant de fait pose les actes suivants: la passation des commandes, la conclusion des emprunts, l'intervention dans la conclusion de contrats, l'organisation de la situation financière de l'entreprise et la négociation avec ses différents partenaires financiers, la tenue de la comptabilité avec détention d'une procuration sur les comptes bancaires de l'entreprise, la fixation des salaires, des primes et promotions, la décision de l'opportunité des investissements et des prises de participations, etc.

Tous ces actes présentent justement des caractéristiques de continuité pour emporter la qualification contre son auteur de dirigeant de fait. En effet, un acte isolé n'ôtant pas aux organes normaux de gestion leurs pouvoirs ne saurait être déterminant pour la qualification de dirigeant de fait57. L'intérêt pour la jurisprudence de se déterminer ainsi est de savoir quelle est la personne susceptible d'engager sa responsabilité pour d'éventuelles fautes de gestion, d'autant plus qu'il n'existe pas de solidarité entre le dirigeant de droit et celui de fait dans la mise en œuvre de cette responsabilité58.

56. Pour l'ensemble de ces questions, lire : A. L. CAPOEN, préc., note 55, p. 304 et suiv.

57. Pour toutes ces considérations, lire : Beauclair NJOYA KAMGA, "Dirigeants sociaux ", dans P.-G. Pougoue (dir.), Encyclopédie du droit OHADA, Lamy, 2011, p. 648 et suiv.

58. Jean-Pierre LEGROS, "Redressement ou liquidation du dirigeant ", (2009) 81 (4) Revue Droit des sociétés 38. 
Même si l'on pourrait penser que le dirigeant de fait puisse intervenir pour poser des actes pouvant contribuer à la bonne marche de la société, il demeure, à tout prendre, une situation à haut risque 59 . Cela vaut pour la société qui ne peut valablement préjuger de la bonne foi d'un acteur de fait, de sorte que l'encourager peut conduire à la ruine de l'entreprise, dès lors qu'il ne sent pas statutairement obligé à une gestion saine; cela vaut également pour le dirigeant étant ou devenu de fait au regard des sanctions qui le guettent. C'est précisément le sens de cette institution du dirigeant de fait qui permet à la société de rattraper les erreurs commises par une personne sans mandat.

\section{§ II- La contribution des situations de fait à l'efficacité du droit des sociétés}

La réception des situations de fait en droit des sociétés ne s'est pas faite sans une finalité utilitaire. En retour justement, les situations de fait contribuent à l'essor du droit des sociétés ${ }^{60}$. On peut dès l'abord entrevoir cet essor dans l'enrichissement quantitatif de la discipline avec ces entités d'un autre genre qui permettent au droit des sociétés d'encadrer les rapports économiques complexes même aménagés de manière malencontreuse par les parties. Mais ce volet contributif peut être marginalisé puisque nous convenons du caractère irrégulier des situations de fait par rapport aux situations juridiques qu'elles singent, encore qu'ici, le reproche pourrait être tempéré, certaines de ces situations bénéficiant d'une consécration législative avec un régime bien construit.

Mais l'essor du droit des sociétés doit davantage être pressenti dans l'efficacité atteinte par ce système qui protège,

59. Philippe Bouchez EL GHOZI, "Les nouveaux risques des directeurs financiers ", (2010) 726 Revue Banque 68; Aurélie VAUDRY, "Dirigeant de fait : une situation à haut risque ", 2007, en ligne : <http:/ /www.netpme.fr/creation-entreprise/1023-dirigeant-fait-situation -haut-risque.html>.

60. Voir par exemple, Jean-Marie De BERMOnd De VAulX, "L'empire des faits et l'émergence de la notion de société ", (1996) 22 Revue Dalloz, 18. 
d'une part, la société et les parties (A) et, d'autre part, les tiers à la société (B).

\section{A- L'efficacité atteinte par la protection de la société et des parties}

Les situations de fait prévues par le droit des sociétés protègent la société et les parties. Il faut, pour s'en rendre compte, se placer sur le terrain de la société qui doit se prévaloir des actes passés lors de sa formation, des actes du dirigeant de fait ou des opérateurs qui recherchent des règles efficaces pour régir leur rapport élaboré sous forme sociétaire, de la concubine qui souhaite bénéficier de son apport dans l'union de fait. La société étant distincte de ses membres, il importe d'examiner séparément sa protection (1) et celles des parties à la société (2).

\section{1- La protection de la société}

La protection de la société par l'admission des situations de fait doit être envisagée aussi bien au plan existentiel (a) qu'au plan patrimonial (b).

\section{a- La protection de la société dans sa réalité existentielle}

Même régulièrement constituée, la société passe par une phase où elle peut être considérée comme situation de fait en tout cas, jusqu'à son immatriculation, condition de sa personnalité morale ${ }^{61}$. Cette phase comprend la société en formation et la société constituée, mais non encore immatriculée, puisqu'à ce stade la société n'est pas encore dotée de la personnalité morale, elle n'est donc pas encore une situation juridique régulière. Les articles 100 et suivants de l'Acte uniforme distinguent justement, à l'intérieur de la période précédant l'immatriculation, entre la société en formation et la société constituée, mais non encore immatriculée.

61. Art. 98 de l'Audsc et Gie. 
L'intérêt de la distinction est de présenter d'une part, pour la société en formation, la situation où la personne morale en devenir n'existe qu'en projet, dans les idées des fondateurs, qui ne deviennent même pas systématiquement associés. D'autre part, s'agissant de la société constituée, mais non encore immatriculée, le stade où elle est déjà matérialisée par la position des actes constitutifs, notamment la souscription, l'évaluation et la réalisation des apports, la publication de la note d'information exigée des sociétés faisant un appel public à l'épargne62, la rédaction et la signature des statuts, etc.

Il demeure toutefois que les deux situations décrivent un état de fait dans lequel se trouve la société en ce moment, car elle n'a pas encore acquis la personnalité morale par l'immatriculation qui la transforme en une situation de droit.

Il se trouve fatalement que dans la chronologie des actes de création d'une société, l'immatriculation au RCCM intervient seulement lorsque tous les actes de constitution ont été accomplis. Elle peut même intervenir jusqu'à six mois après le dépôt des fonds dans un compte ouvert au nom de la société en formation ou chez un notaire63. Il s'ensuit que la totalité des actes de constitution de la société est posée sous l'empire des faits ${ }^{64}$, de sorte que l'immatriculation n'est qu'un acte déclaratif et non constitutif de l'existence de la société. Celle-ci remonte donc au jour des actes de constitution et même ceux de formation.

62. Art. 86 et suivants de l'Audsc et Gie. Sur cette note, instruction Générale $\mathrm{n}^{\circ}$ 002/CMF/04 relative à la note d'information exigée des émetteurs faisant un appel public à l'épargne (en application de la loi 99/015 du 22 décembre 1999 portant création et organisation d'un marché financier au Cameroun. Voir Jean GATSI, "Le marché financier au Cameroun ", (2001) 45 Juridis périodique 63); également, art. 825 du même Acte uniforme.

63. Art. 314, al. 3 pour la SARL, art. 393 pour la SA, mais 399 et suivants pour la SA constituée avec apport en nature ou autres avantages particuliers.

64. J.-M. DE BERMOND De VAulX, préc., note 60, 18. 
Le fait que le droit des sociétés ne combatte pas la situation de fait dans laquelle se trouve la société avant son immatriculation la conforte dans son existence. En réalité, pour que la société existe, il faut ce passage obligatoire par la situation de fait où tous les actes concourant à sa mise sur pied sont accomplis. S'il fallait ne pas tenir compte des actes posés à ce stade du fait que la société n'a pas encore acquis la personnalité juridique, l'existence de la société en serait compromise. Cette période revêt même une importance capitale pour les pouvoirs publics qui exercent un contrôle sur les actes pris au nom de la société en formation ou la société constituée, mais non encore immatriculée, afin que la personne morale puisse partir sur des bases réelles. En effet, des conditions minimales sont exigées pour que la société soit réputée continuer avec les actes accomplis pour elle sous l'empire des faits ${ }^{65}$. Ces conditions ne ressortent pas clairement de la lecture de la loi, mais de l'office du juge. Il faut, pour que la société immatriculée reprenne les actes accomplis pour elle au jour de sa formation ou de sa constitution, que lesdits actes aient été nécessaires et utiles. Cela veut dire qu'ils aient été posés dans l'intérêt de la société et que cet intérêt puisse être justifié. Dans le cas contraire, si les fondateurs ont accompli des actes ruineux, et là, la société est en droit de les rejeter, ils engagent leur responsabilité solidaire et indéfinie pour les obligations qu'ils comportent ${ }^{66}$. En revanche, lorsque la société a accepté reprendre un acte à son compte, même si celui-ci a dépassé les pouvoirs des mandataires commis à cet effet, ledit acte est réputé avoir été accompli de manière utile ${ }^{67}$.

65. Voir Richard CRÔNE, "Conditions de reprises des engagements d'une personne ayant agi au nom de la société en formation ", Note sous Cass. civ. $1^{\text {re }}, 26$ avril 2000, I, n 123, p. 82; Rép. Defrénois, 2001, n 9, p. 569; Corinne BOISMAIN, "Reprise des engagements d'une SARL en formation : ratification par un mandat postérieur à l'acte ", (2008) 43 Semaine Juridique, $\mathrm{n}^{\circ}$ 2299, p. 29-32.

66. Art. 110 al. 2 de l'Audsc et Gie.

67. Marie-Laure Coquelet, "Validité et modalités de la reprise de l'enchère faite au nom d'une société en formation ", (2009) 12 Revue Droit des sociétés, commentaire $\mathrm{n}^{\circ} 217$, p. 12-13, note sous Cass. civ., 2e 10 septembre 2009. 
Un tel contrôle exercé par les pouvoirs publics permet d'éviter que le patrimoine de la société nouveau-né ne soit pas anormalement grevé d'un passif, ce qui nuirait fatalement à son existence future.

\section{b- La protection de la société dans sa réalité patrimoniale}

En prenant en compte la réalité que constituent les situations de fait, le droit des sociétés met en place un système de protection du patrimoine de la société commerciale. Les développements précédents ont été consacrés à la période avant l'acquisition de la personnalité morale, et au cours de laquelle le capital social est constitué par les souscriptions de parts suivies de leur libération. Point n'est besoin d'y revenir ici. Seule nous intéressera, dans ce paragraphe, la protection du patrimoine courant un risque du fait d'une situation de fait. À ce titre, l'encadrement du dirigeant de fait fournit d'importants éléments d'analyse de la situation. En effet, les actes posés par un dirigeant de fait peuvent avoir deux effets, tous importants pour le droit des sociétés.

D'une part, les actes peuvent être ruineux, c'est-à-dire de nature à compromettre le patrimoine de la société. Il est évident que l'un des risques importants courus par la société commerciale est l'atteinte à son patrimoine par les personnes qui y exercent une fonction de direction du fait de leur position privilégiée. Le problème ne se pose pas s'agissant du dirigeant de droit; le droit des sociétés qui l'a suffisamment réglementé a justement prévu un régime pour sanctionner ses fautes de gestion68. Dans cette optique, il faut voir comme une marque d'efficacité le fait d'étendre le régime de sanction du dirigeant de droit au dirigeant de fait qui est une situation de fait.

68. Le dirigeant de droit est, par exemple, aux termes de l'article 891 de l'Audsc et Gie, passible des poursuites pour abus des biens et du crédit de la société. 
En effet, le système mis en place par le droit des sociétés permet, au regard de la théorie générale du droit, d'attribuer d'abord à cette personne qui s'est irrégulièrement immiscée dans la gestion de la société la qualification de dirigeant. Le dirigeant de fait est avant tout un dirigeant, cela permet donc de lui appliquer les règles prévues pour les fautes de gestion d'un dirigeant de droit69.

C'est en prolongeant ce souci que le législateur OHADA des procédures collectives prévoit l'extension des procédures collectives au dirigeant même de fait ${ }^{70}$. L'article 180 de l'Acte uniforme relatif aux procédures collectives d'apurement du passif énonce justement "[1]es dispositions du présent chapitre sont applicables, en cas de cessation des paiements d'une personne morale, aux dirigeants de personnes physiques ou morales, de droit ou de fait, apparents ou occultes, rémunérés ou non et aux personnes physiques représentants permanents des personnes morales dirigeantes". Ce texte fait référence au chapitre VI du Titre II et porte des dispositions particulières aux dirigeants de personnes morales. Les dispositions dont il s'agit sont le comblement du passif et l'extension des procédures collectives. Cela veut dire que le dirigeant de fait peut être condamné au comblement du passif ${ }^{71}$, tout comme il peut lui être étendue la procédure collective ouverte contre la personne morale au sein de laquelle il a posé des actes positifs de gestion ${ }^{72}$. Le Titre III va plus loin pour prévoir le prononcé de la faillite personnelle du dirigeant de fait. Pour toutes ces hypothèses, il subit le même sort que le dirigeant de droit.

69. А. АКАм АКАм, préc., note 51, 214 et 234.

70. Art. 180 Aupc.

71. Art. 183 Aupc.

72. Art. 189 Aupc. Voir, Filiga Michel SAWADOGO, "Sanctions civiles et commerciales des procédures collectives d'apurement du passif ", dans P.-G. Pougoue, Encyclopédie du droit OHADA, Lamy, 2011, p. 1805. 
C'est ainsi qu'il peut être condamné au comblement du passif de la société73 dès que certaines conditions sont réunies, à savoir l'existence d'une faute de gestion, la preuve d'une insuffisance d'actif et un lien de causalité entre la faute et le préjudice de la société74. Il n'y a pas de définition légale de la faute de gestion. Toutefois, on peut considérer que c'est une faute dans la gestion de la société se caractérisant, par exemple, par des initiatives irréfléchies, l'incurie ou la négligence dans les opérations, la poursuite abusive d'une exploitation déficitaire, le défaut de réaction alors qu'une alerte a été déclenchée, la nontenue d'une comptabilité ou sa mauvaise tenue, toute chose résultant à un préjudice pour la société ${ }^{75}$.

Dans la même perspective, il peut lui être étendue la procédure collective ouverte contre la personne morale. Cette sanction dépasse le cadre de l'obligation de combler le passif. En effet, pour cette dernière, le dirigeant peut n'être condamné qu'à supporter une partie du passif, en tout cas, la partie qui est imputable à sa faute de gestion. L'extension de la procédure collective se révèle être plus grave, car ici, l'obligation du dirigeant comprend, outre son propre passif, celui de la personne morale. Quatre conditions sont nécessaires pour cette sanction ${ }^{76}$, dont les trois premières décrivent une confusion de son patrimoine par le dirigeant, avec celui de la société. Il s'agit, en premier lieu, de l'exercice d'une activité commerciale personnelle, soit par personne interposée, soit sous le couvert d'une personne morale masquant ses agissements; en second lieu, du fait de disposer du

73. Art. 180 et 183 de l'Acte uniforme portant organisation des procédures collectives d'apurement du passif. Voir, A. AKAM AKAM, préc., note 51, 234; En droit comparé : Cass. com. 9 novembre 1993, Dalloz 1995, sommaires commentés, p. 79; voir B. FEUGERE, "Le dirigeant d'une personne morale cité devant le tribunal de commerce pour comblement du passif (Article 180 de la loi du 25 janvier 1985) ", (1999) RJ com. 333.

74. A. АKAM АKAM, préc., note 51, 235 et suiv.

75. Pour toutes ces questions: F. M. SAWAdogo, "Sanctions civiles et commerciales des procédures collectives d'apurement du passif ", préc., note 72 , p. 1805 .

76. Art. 189 de l'Acte uniforme portant organisation des procédures collectives d'apurement du passif. 
crédit ou des biens de la personne morale comme des siens propres; en troisième lieu, de la poursuite abusive par le dirigeant social, dans son intérêt personnel, d'une activité déficitaire qui ne pouvait conduire qu'à la cessation des paiements de la personne morale. La quatrième condition vient compléter l'obligation de combler le passif et se réalise lorsque le dirigeant soumis à cette condamnation ne s'acquitte pas de son montant ${ }^{77}$.

En outre, il peut être tenu de supporter ses engagements à l'égard des tiers. En règle générale du droit des sociétés, lorsque le dirigeant social a posé des actes qui ne relèvent pas de l'objet social, il engage sa responsabilité personnelle à l'égard des tiers si surtout la société prouve l'existence d'une collusion frauduleuse entre ces tiers et le dirigeant78. C'est l'application du principe jurisprudentiel de la faute détachable des fonctions ${ }^{79}$. Cette règle est extensible au dirigeant de fait dans la perspective de la protection du patrimoine de la société.

D'autre part, les actes du dirigeant de fait pourraient être bénéfiques pour la société, même s'il demeure évident que le dirigeant de fait est une situation à haut risque ${ }^{80}$. Dans ce cas, il n'est point tenu compte de sa qualité de dirigeant de fait, les actes sont purement et simplement repris par la société à son compte comme s'ils avaient été posés par un dirigeant régulier. Le droit des sociétés ne sanctionne donc la direction de fait que lorsqu'il y a un acte de gestion ayant entamé le patrimoine de la société. En

77. Pour toutes ces questions : F. M.SAWADOGO, préc., note 72, p. 1805.

78. Art. 122,330 et 740 de l'Audsc et Gie.

79. Sur la définition de la notion, l'on peut emprunter au juge Français dont le système est proche de l'Ohada : Cass. com., 20 mai 2003, pourvoi ${ }^{\circ}$ 99-17092, Bull. 2003, IV, n 84 p. 94; voir, MASCRE HEGRY et al., "Responsabilité personnelle des dirigeants sociaux: Une nouvelle définition de la faute séparable des fonctions? ", Mascré Hegry associés, octobre 2003, en ligne : <www.mascre-hegry.com>; Bénédicte BouviER, "La responsabilité du dirigeant pour faute "séparable de l'exercice de ses fonctions" ", en ligne : <http://capitalinvest.gramond.pro>, 9 février 2011 (consulté le 26 mars 2012); A. AKAM AKAM, préc., note 51, 228; Gilles AUZERO, "L'application de la notion de faute personnelle détachable des fonctions en droit privé ", (1998) Revue Dalloz affaires 502.

80. Ph. Bouchez El GHOzI, préc., note 59, p. 68; A.VAudrY, préc., note 68. 
effet, l'article 300 de l'Acte uniforme (à propos de la société en commandite simple) qui donne la référence au dirigeant de fait permet de le penser dès le moment qu'il ne vise l'engagement de la responsabilité du commanditaire ayant participé à la gestion que pour le passif résultant de cette gestion et non de l'actif. Il n'y a pas un régime de sanction du simple chef de la gestion de fait, mais du résultat préjudiciable à la société 81 .

\section{2- La protection des parties}

La notion de parties doit être entendue dans le sens de ceux qui participent à la situation de fait. Leur protection sera alors envisagée dans le cadre d'une société de fait qui, comme nous allons l'observer, n'est pas négligeable. En effet, l'un des mérites de l'accueil des sociétés de fait réside dans l'aménagement des rapports des parties. La reconnaissance d'une société de fait entre des personnes permet justement de les départager.

Mais plus qu'un simple calcul arithmétique, il s'agit au plan de la théorie générale du droit de la possibilité d'appliquer les règles du droit des sociétés à des situations qui n'en remplissent pas les conditions. Cela constitue un avantage de cette discipline qui réussit à encadrer les relations d'affaires des parties, même formées de manière malencontreuse.

Il s'agira donc de liquider la société reconnue de fait entre les parties ${ }^{82}$, afin que chacune puisse retrouver son investissement. Liquider dans ce contexte signifie partager ${ }^{83}$. C'est que la société est l'un des rares modèles d'organisations où les

81. Voir par exemple, Renaud SALOMON, "Les conditions de la répression du dirigeant de fait en droit pénal des sociétés ", (2004) 11 Revue Droit des sociétés, 34-36.

82. En marge de la volonté de créer une société de fait manifestée par les parties et que nous avons explorée en amont, c'est la sanction du juge quant à la nature de la société qui est déterminante. C'est au juge qu'il revient en fait de décider s'il y a ou non société de fait et d'en tirer les conséquences.

83. Voir, Jean Herall, Pratique des liquidations-partages, Paris, Litec, 1999. 
intérêts des parties sont communs et non opposés ${ }^{84}$; c'est ce qui va justifier le partage, entre associés de fait, du patrimoine de la société. Le juge va donc soit ordonner le partage du patrimoine commun en nature, soit en ordonner la vente pour la distribution du produit net ${ }^{85}$. Partager le patrimoine doit s'entendre de son actif et de son passif. L'accueil des situations de fait est surtout intéressant, car dans le cas de la société de fait, un régime particulier a été prévu par rapport aux effets quant aux droits des parties. Ce régime est celui de la société en nom collectif. Le liquidateur nommé va donc payer les créanciers et répartir entre les associés de fait le solde disponible 86.

Une telle technique est intéressante entre concubins ${ }^{87}$. L'ensemble des législations des pays membres de l'OHADA ne connaît pas un régime juridique du concubinage. Cette situation est même combattue par le droit qui encourage le mariage civil, en tout cas, le mariage légal, que ce soit selon la coutume ou selon les lois civiles. À l'heure actuelle, s'agissant des droits pécuniaires des concubins en fin d'union, cet écueil peut être réputé corrigé par l'institution de la société en participation. Si les concubins créent une telle société, le problème de preuve de leur participation dans une affaire commune ne se posera pas, l'existence même de la société en participation se prouvant par tout moyen ${ }^{88}$. Pareille rigueur n'est pas imposée à la femme mariée délaissée qui pourtant peut être moins patrimonialement active qu'une concubine amoureuse.

84. Voir, Dominique SCHMIDT, "De l'intérêt commun des associés ", (1994) I JCP 3793; Paul DIDIER, "Le consentement sans l'échange, contrat de société dans l'échange des consentements ", (1995) Rev. Jurisp. Com. 74; Yves GuYon, "La fraternité dans le contrat de société ", (1989) Revue des sociétés 439.

85. Cass. com. 16 juin 1998, pourvoi $\mathrm{n}^{\circ}$ 96-12337, Bull. 1998, IV, $\mathrm{n}^{\circ}$ 203, p. 168.

86. Art. 223 et suiv.; particulièrement, 231 de l'Audsc et Gie.

87. Voir Audrey MELlac, "Techniques de droit commun applicables à la rupture du concubinage et du PACS ", master II recherche, Strasbourg, Université Robert Schuman, 2007, p. 36.

88. La société en participation est le type d'organisation qui permettrait aux concubins d'aménager leurs rapports sans devoir s'encombrer du formalisme du droit des sociétés. 
À défaut d'une société en participation, et cela vaut pour les opérateurs économiques qui n'auront pas pris la peine de formaliser leur relation, le droit des sociétés révèle son efficacité en fournissant aux juges les moyens de régler les rapports des parties. Les juges peuvent ainsi recourir à la technique de la société créée de fait afin de liquider le patrimoine des parties. Le problème s'est justement posé en jurisprudence assez récente où les juges ont pu fixer conditions de la reconnaissance d'une société créée de fait entre des parties ${ }^{89}$. La CCJA a même été sollicitée à propos d'une affaire pareille; malheureusement, à cause d'un vice de procédure, elle n'a pas eu l'occasion de se prononcer sur les mérites du pourvoi ${ }^{90}$.

La précision des conditions de qualification en société créée de fait est tout à fait révélatrice de la possibilité du recours à la notion de société créée de fait et de l'efficacité du droit des sociétés à aménager les rapports de fait des parties, même à l'égard des tiers.

\section{B- L'efficacité atteinte par la protection des tiers}

L'essor du droit des sociétés s'affirme au travers des situations de fait, particulièrement sur les droits des tiers. La technique de la société de fait joue encore un rôle important ici. Il est en effet important pour un tiers que soit reconnue l'existence d'une société créée de fait entre des personnes qui on traité avec lui, pour les voir tous participer à l'exécution de l'obligation née de leur activité à son profit. Le système est tout à fait intéressant en ce qu'il donne le droit au tiers d'intenter une action en vue de la reconnaissance d'une société de fait entre ses débiteurs (1); cette

89. Par exemple, CA Centre, Arrêt n ${ }^{\circ}$ 380/CIV du 05 Novembre 2008 : KaGO LElE J. c/ TCHOUPO Ch., Ohadata J-10-134, www.ohada.com; CA Ouagadougou, Arrêt n 86 du 21 Avril 2006 : ADOKo Sessinou Bernard c/ Nacoulma Désiré, Yanogo B. Michael, Songnaba / Compaore Claudine, en ligne : <www.ohada.com> (consultés le 18 Février 2013).

90. Boubakar DiAllo, "La société créée de fait dans l'OHADA ", note sous CCJA n 31, 04 novembre 2004 : Ayant droit de B. c/ Madame A ", Rev. Jurifis Infos, $\mathrm{n}^{\circ}$ 2, Mars-Avril, 2009, chron. OHADA, p. 18. 
action aboutit à l'application de la responsabilité solidaire des associés en nom collectif (2).

\section{1- L'existence d'une action en reconnaissance de société de fait au profit du tiers}

En vue de la reconnaissance d'une société de fait entre des personnes, le tiers dispose d'une action en justice. Cette action est fondée sur l'article 866 de l'Acte uniforme relatif au droit des sociétés qui dispose "[q]uiconque y ayant un intérêt peut demander à la juridiction compétente du lieu principal de l'activité d'une société de fait, la reconnaissance de la société de fait entre deux ou plusieurs personnes dont il lui appartient d'apporter l'identité ou la dénomination sociale "91. Par l'usage du pronom quiconque, le législateur laisse ainsi suggérer que l'action est ouverte à toute personne. Il suffit que le demandeur à cette action puisse justifier d'un intérêt. Le tiers peut être présumé avoir un intérêt à voir déclarer l'existence d'une société de fait entre ses débiteurs, lorsque la créance qu'il détient contre eux est née d'une activité à laquelle chacun d'eux a participé. Ainsi, comme cela est notoirement connu, l'intérêt à agir en justice désigne le profit, l'utilité ou l'avantage que l'action est susceptible de procurer au plaideur92. La reconnaissance d'une société de fait entre ses débiteurs lui permettra à coup sûr de bénéficier des règles de la responsabilité indéfinie et solidaire des associés en nom ainsi que le prévoit l'article 868 de l'Acte uniforme. Cette application est de nature à favoriser le recouvrement efficace de sa créance.

Doit-on réfléchir sur sa qualité? Les deux notions de qualité et d'intérêt s'imposent en effet comme conditions sine qua non de

91. CA Ouagadougou, Arrêt n 86 du 21 avril 2006, préc.

92. Voir J. GATsI, Nouveau dictionnaire juridique, préc., note 2, p. 181; Gilbert KERE KERE, Droit civil processuel. La pratique judiciaire au Cameroun et devant la CCJA, SOPECAM, 2006, p. 19; Loïc CADIET et Emmanuel JEuland, Droit judiciaire privé, Lexis Nexis, Paris, Litec, 2006, p. 218; Louis GARAUD, "L'intérêt pour agir en justice. Contribution à la notion d'intérêt en droit positif " thèse de doctorat, Poitiers, Université de Poitiers, 1959. 
l'action en justice ${ }^{93}$. La qualité est le titre juridique qui confère le droit d'agir, c'est-à-dire de solliciter du juge qu'il examine le bienfondé d'une prétention ${ }^{94}$. Le système anglo-saxon ne distingue certainement pas entre intérêt et qualité à agir ${ }^{95}$. Les deux notions se retrouveraient peut-être dans l'expression de cause of action qu'un juge a définie comme étant : "[e]very fact which must be proved if traversed to support his right and the judgment of the court ". Une autre définition a été fournie pour dire : "[t]he factual situation the evidence of which justifies a person to obtain from a court a remedy against another person "96. Elle se déduit souvent de l'intérêt, notamment si le tiers intente une action personnelle fondée sur un intérêt né et actuel. Les auteurs pour caractériser cette induction se situent dans les actions banales ${ }^{97}$. Il va de soi que dans les actions dites complexes, notamment où l'intérêt peut ne pas être direct, la qualité devra être justifiée indépendamment de l'intérêt. Le tiers justifiant d'un intérêt personnel à agir a donc la qualité pour le faire.

Il ne lui restera qu'à rapporter la preuve dont les conditions pas faciles ont été facilitées en jurisprudence. Les juges accordent, par exemple, aux créanciers de fonder leur action sur l'apparence de l'existence de la société de fait. Cette apparence s'apprécie globalement, indépendamment de l'existence apparente de chacun

93. Art. 2 et suivants du Code de procédure civile et commercial: CS, Arrêt $\mathrm{n}^{\circ}$ 50/CC du 18 janvier 1982, dans S. NGUE, Code de procédure civile et commerciale, Yaoundé, MINOS, 2001, p. 9; CSCO, Arrêt n ${ }^{\circ} 103 / \mathrm{C}$ du 7 avril 1970, BACS, n² 22, p. 2691.

94. G. Kere Kere, préc., note 92; L. CADiet et E.Jeuland, préc., note 92, p. 224.

95. Le Cameroun a la particularité d'être bi-jurial pour avoir subi les deux colonisations française et anglaise et donc a hérité aussi bien de la tradition civiliste que de celle d'inspiration common law. Voir Roger Sockeng, Les institutions judiciaires au Cameroun, Macacos, 2005, p. 5 et suiv..

96. Pour l'ensemble de ces questions, lire Michael Akomaye YANou, Practice and procedure matters in the courts of records in Anglophone Cameroon, Wusen Publishers, 2012, p. 21.

97. L. CADIET et E.JEuland, préc., note 92, p. 225. 
des éléments constitutifs de la société98. La loi elle-même a permis de déduire l'existence de la société de fait du comportement des associés de fait ${ }^{99}$. Évidemment, la théorie de l'apparence ne bénéficie qu'aux tiers demandeurs à l'action en reconnaissance de société de fait. Les parties n'y sont pas admises. Il leur est exigé de rapporter la preuve de l'existence matérielle des éléments constitutifs d'une société. En effet, la partie qui demande la reconnaissance de l'existence d'une société de fait entre elle et son partenaire devra prouver l'existence d'apports de chacun, la volonté de collaborer sur un pied d'égalité, la volonté de contribuer aux pertes et celle de partager les bénéfices.

S'agissant des moyens de preuve recevable au bénéfice du tiers comme celui de parties d'ailleurs, la loi précise que "[1]'existence de la société de fait est prouvée par tout moyen "100. Lorsque le juge reconnaît l'existence de la société de fait, les règles de la société en nom collectif sont appliquées aux associés.

\section{2- L'application aux associés de fait de la solidarité de la société en nom collectif}

L'intérêt de la reconnaissance de l'existence d'une société de fait entre des personnes est pour le tiers de les voir soumis aux règles de la société en nom collectif ${ }^{101}$. Cette applicabilité du droit des sociétés appelle au moins deux observations.

98. De manière générale, on peut emprunter cette précision au juge français : Cass. com., 29 mars 1994, Bull. Joly, 1994, p. 665, note J. Vallansan; Cass.com 8 juillet 2003, pourvoi $n^{\circ}$ 99-19821; Cass.com 8 juillet 2003, pourvoi $\mathrm{n}^{\circ}$ 02-12035, courdecassation.fr, lire Michel DE GAUDEMARIS, "Théorie de l'apparence et sociétés ", (1991) Revue des sociétés 465.

99. Art. 864 de l'Audsc et Gie; CA Ouagadougou, ch. civ. \& com., n 86, 21 avril 2006, préc., www.ohada.com, Ohadata J-09-24; CA Daloa ${ }^{\circ}$ 195/2000 du 24 mai 2000 : WAN KIL LEE c/ JEON KUK HYUN, préc., note 35.

100. Art. 867 de l'Audsc et Gie : CA Centre, Arrêt n ${ }^{\circ} 380 /$ CIV du 05 Novembre 2008, préc., Ohadata J-10-134, www.ohada.com; CA Ouagadougou, Arrêt n ${ }^{\circ} 86$ du 21 avril 2006, préc.

101. Art. 868 de l'Audsc et Gie. 
D'une part, elle révèle l'attractivité de ce corps de règles qui intègre dans son champ d'application les situations non conformes, pour la plupart, à ses exigences, afin de leur faire produire des conséquences juridiques. Tel auteur pourrait y voir une marque de la bonté du législateur ${ }^{102}$ qui prête ainsi son office pour l'encadrement des situations de fait. Tel autre y verrait une marque de simplification du droit des sociétés ${ }^{103}$ qui se départit ainsi de sa trop grande propension au formalisme. Tout cela a, pour nous, un but d'efficacité du système.

D'autre part, le choix des règles qui combinent le droit des obligations avec le système spécifique du droit de sociétés renforce cette efficacité. Le recours aux dispositions applicables en matière de société en nom collectif en est justement le signe. Quelle est la particularité de ces règles? La particularité de la société en nom collectif est justement que les associés sont indéfiniment et solidairement responsables des dettes sociales ${ }^{104}$. Il est peut-être inadapté de parler, s'agissant d'une société de fait, de responsabilité des dettes sociales. En effet, la plupart du temps, les engagements n'ont été contractés que par une seule personne, même si les autres en ont profité, et puis la société n'ayant pas d'existence ostensible n'a pas pu agir personnellement. La reconnaissance de l'existence de la société de fait a pour effet de postuler une fiction de la personnalité morale à ce groupe et faire agir les règles du droit des sociétés.

Concrètement, chacun des associés de fait va devoir participer à la dette contractée par l'un d'eux. Le tiers aura donc la possibilité de s'adresser à l'un quelconque d'entre eux pour le tout, jusqu'à pouvoir pratiquer l'exécution forcée sur ses biens. Évidemment, la décision qui reconnaît l'existence d'une société de fait entre des personnes condamne celles-ci à répondre solidairement des engagements de cette société. Après leur avoir signifié la décision pour faire courir les délais de recours, le tiers

102. Gérard CoRnu, "La bonté du législateur ", (1991) 90 (2) RTD. Civ. 283.

103. Bernard SAINTOURENS, "La simplification du droit des sociétés ", (1994) RIDC 93.

104. Art. 270 de l'Audsc et Gie. 
peut, si sa créance est menacée, pratiquer d'abord une saisie conservatoire sur les biens de celui auquel il s'est adressé. Dès l'obtention de la grosse du jugement, il ne lui reste qu'à transformer sa saisie conservatoire en saisie-exécution. Ce système ne peut que conforter l'idée selon laquelle la réception des situations de fait en droit des sociétés est un gage de l'efficacité de cette discipline.

\section{CONCLUSION}

$\mathrm{Au}$ terme de cette étude, l'enseignement que l'on peut retenir, en substance, est que le droit des sociétés et les situations de fait font plutôt bon ménage. L'abondance de ces catégories "juridiques "105 dans le droit des sociétés se justifie par les finalités qu'elles permettent d'atteindre. En effet, en adoptant les situations de fait comme des entités créatrices de droit, ce qui est original en théorie du droit, la discipline atteint l'efficacité qui en est attendue, s'agissant de l'encadrement des relations d'affaires et de la protection de certaines parties faibles.

Le droit des sociétés prend ainsi à profit la violation des règles de formation des institutions du droit pour en retirer un minimum de substance nécessaire à la satisfaction des parties, des tiers et même de la société commerciale. La construction du dirigeant de fait permet justement de protéger la société des fautes de gestion d'une personne ayant posé des actes positifs de gestion alors qu'elle était dépourvue de mandat pour l'administrer ${ }^{106}$. De même, l'appartenance d'une société à un groupe peut contribuer à sa croissance. L'existence de la société en participation permet aux parties de bénéficier des règles du droit des sociétés sans avoir à s'en embarrasser du formalisme. La reconnaissance de

105. Nous osons employer l'expression "catégorie juridique " parce que les situations de fait, au-delà de tout, sont reconnues par le droit des sociétés, ne serait-ce que dans l'optique d'en tirer les conséquences exposées dans la présente étude.

106. A. AKAM AKAM, préc., note 51, 234; L. CAPOEN, "La responsabilité du banquier à l'égard des entreprises en difficultés ", op. cit., p. 304 et suiv. 
l'existence d'une société de fait permet de protéger aussi bien les associés de fait que les tiers.

Toutes ces considérations font assurément du droit des sociétés un droit adapté aux besoins des entreprises. Sa flexibilité107 à l'égard de certaines catégories juridiques participe d'un souci de simplification 108 permettant aux destinataires de la législation sur les sociétés commerciales d'en tirer le plus grand bénéfice.

L'on peut regretter l'incertitude entretenue par le législateur quant à la différence à établir par exemple entre société de fait et société créée de fait ${ }^{109}$, mais celle-ci est corrigée par la jurisprudence qui, elle, emploie les deux expressions indifféremment pour caractériser la situation de fait. Cela n'exclut cependant pas une révision législative à l'effet de clarifier cette distinction.

107. Sur l'expression, Bernard SAInTOuRens, "La flexibilité du droit des sociétés ", (1987) RTD. Com. 455.

108. Sur l'expression, B.SAINTOURENS, préc., note 103..

109. Sur la question Ch. MBA-OwONO, préc., note 25, p. 467 et suiv.; Ohadata D-10-19. 
